\title{
Diagnóstico del síndrome de Bartter por estudio sistemático de la hipokalemia
}

\author{
Dres. Raúl Femández $S{ }^{1}{ }^{1}$ José A. Rodriguez P. ${ }^{2}$ Francisco Barrera $Q^{3}$ Jorge Weinberger K. ${ }^{4}$
}

Bartter's syndrome: Diagnosis by systematic aproach to hy pokalemia

\begin{abstract}
The case of a 4 year-old female patient with persistent and severe hypokalemia, metabolic alkalosis, hyperreninemia with hyperplasia of the juxtaglomerular apparatus and nomal blood pressure is presented. A systematic search for other possible causes of hypokalemia was undertaken. A decrease in renal fractional distal chloride reabsorption comparable to that found in other patients with Bartter's syndrome, allows a rational explanation for the development of her signs and symptorus.
\end{abstract}

Dentro de las causas de hipokalemia importante y mantenida debe considerarse el sindrome de Bartter, que a pesar de ser un diagnóstico poco frecuente es susceptible de un enfoque terapéutico que puede mejorar ostensiblemente el trastorno metabólico., ${ }^{1,2,3}$

En la descripción original de este sindrome, ${ }^{4}$ Bartter consideró la existencia de hipokalemia con alcalosis metabólica, hiperplasia del aparato yuxtaglomerular con hiperreninemia e hiperaldosteronismo secundario con presión arterial normal. Ultimamente se le atribuye importancia a alteraciones en el transporte activo renal de cloro como factor etiológico primario. ${ }^{\overline{7}}$

A continuación se describe el caso de una preescolar en que se llegó al diagnosticó mediante el estudio sistemático de su hipokalemia y se comentan las diferencias entre el síndrome de Bartter y cuadros clínicos similares.

\section{CASO CLINICO}

P.P.Q., pre-escolar de 4 años 6 meses de edad,

\footnotetext{
1 Medico becario, Servicio de Pediatria, Hospital Paula Jaraquemada.

2 Departamento de Enférmedades Metahólicas, Endocrínolópicas y Reumatológicas, Facultad de Medicina, Pontificia Universidad Católica de Chile.

${ }^{3}$ Departamento de Pediatria y Cinugia Infantil Central, Facultad de Medicina, Lniversidad de Chile, Servicio de Pediatría, Hospital Paula Jaraquemada.

${ }^{4}$ Director Medicina Experimental, Facultad de Medicina, Uníversidad de Chile, División Sur.
}

antecedentes de padres sanos no consanguíneos, hija úmica. Proviene de un embarazo que cursó con prurito gravídico en su tercer trímestre.

Parto de término, pesó al nacer: $3.500 \mathrm{~g}$. con aumento normal hasta los 3 meses de vida. A los 4 meses inicia desnutrición llegando a Grado III a los 11 meses. Se descartó desnutrición primaria, enteroparasitosis, infección urinaria, enfermedad celíaca.

Ingresó al Servicio de Pediatría del Hospital Paula Jaraquemada a los 3 años 7 meses de edad con deshidratación grave y marcado compromiso de conciencia. A pesar de ello tenía poliuria de más de $120 \mathrm{ml} / \mathrm{kg} / \mathrm{día}$ a lo que inicialmente se atribuyó la deshidratación.

En el coprocultivo se aisló Shigella Flexneri, en el urocultivo, más de 100.000 col. de E. Coli $x$ $\mathrm{mm}^{3}$ y en el sedimento urinario habían piocitos y placas de pus abundantes. La pielografía endovenosa mostró sombras renales de forma y tamaño normal con dilatación marcada de ambos uréteres.

La paciente presentaba alcalosis metabólica hipokalémica con hiponatremia, hipocloremia y presión arterial normal. Pasado el cuadro agudo evolucionó ambulatoriamente por 1 año con suplementación de $8 \mathrm{~g}$ de $\mathrm{NaCl}$ y $5 \mathrm{~g}$ de $\mathrm{KCl}$ al día.

Rehospitalizada a los 4 años 6 meses de edad se observó pre-escolar de mejor aspecto general. Peso: $10,6 \mathrm{~kg}$ (percentil $<3$ ), talla: $87 \mathrm{~cm}$ (percentil $<3$ ), P.C.: $47 \mathrm{cms}$ (percentil $<3$ ), presión arterial: normal $(85 / 46 \mathrm{~mm} \mathrm{Hg})$.

Había marcada atrofia de masas musculares y de 
tejido adiposo, hipotonía muscular y fatigabilidad fácil e hiporreflexia osteotendínea. Coeficiente de desarrollo para 3 años. Edad ósea 3 años. Sedimento urinario: nomai.

\section{METODOS DE ESTUDIO}

E] sodio y potusio en plasma y orina se determinaron por fotometria de llama; el cloro por $\mathbf{t}$ tulación. E] $\mathrm{CO}_{2}$ total y $\mathrm{pH}$ por medio de un $\mathrm{pH} / \mathbf{B l o o d}$ Gas Analyzer. El nitrógeno ureíco por el método de masa y reacción de Berthelot. La creatinina en plasma y orina con Technicon Auto Analyzer. La aldosterona urinaria se midió por radioinmunoanálisis del compuesto ácido lábil a $\mathrm{pH}^{6}{ }^{6}$

La actividad de renina en plasma se midió por radioinmunoanálisis como concentración de angiotensina I, generada en una hora de incubación con "kit" de New England Nuclear. Los 17 hidroxiesteroides urinarios se deteminaron según la técnica de Porter y Silber. ${ }^{7}$ Los 17 ketoesteroides urinarios se determinaron según la técnica de Zimmermann. ${ }^{8}$

La osmolalidad plasmática y urinaria se midió determinando el descenso del punto de congelación.

Las resultados se expresan como promedio \pm error standard.

\section{RESULTADOS}

Los valores de electrolitos plasmáticos y urinarios, $\mathrm{pH}$ y $\mathrm{CO}_{2}$ en plasma, nitrógeno ureico, actividad de renina plasmatica, aldosterona, $\mathrm{pH}$ y volumen urinarius se muestran en la Tabla $l$.

La depuración de creatinina fue de 128 $\mathrm{ml} / \mathrm{min} / 1.73 \mathrm{~m}^{2}$. Los 17 hidroxiesteroides en orina de 24 horas fluctuaron entre 1.0 y $2.6 \mathrm{mg} / 24$ horas y los 17 keteosteroides variaron entre 0.8 y 1.8 mg/24 horas. El magnesio plasmático fue de 1.42 $\mathrm{mEq} / \mathrm{l}$.

Se efectuó infusión de Angiotensina II (Hipertensina CIBA) y noradrenalina, observándose que para producir un aumento de $20 \mathrm{~mm} \mathrm{Hg}$ en la presión diastólica, se necesitó infundir 135 $\mathrm{ng} / \mathrm{kg} / \mathrm{min}$ de angiotensina II y $400 \mathrm{ng} / \mathrm{kg} / \mathrm{min}$ de noradrenalina, siendo lo normal hasta 10 $\mathrm{ng} / \mathrm{kg} / \mathrm{min}$ de angiotensina II y $50 \mathrm{ng} / \mathrm{kg} / \mathrm{min} \mathrm{de}$ noradrenalina (Figura 1).

A continuación, en equilibrio con una dieta es-
HALA -1

SINDROME DE BARTTER, ESTUDD METABOLICO

INGESTA PROMEOLO:(mEq/kg/dia)

Na: $4,7 \quad K: 2,8-4,5$

Agua: (m/ $/ \mathrm{kg} / \mathrm{dia}): 162.7$

Calorias:(cal/hg/dia):90,36

\begin{tabular}{|c|c|c|c|}
\hline n & RASMAA & ₹ & E.L \\
\hline 7 & $n \in m \in q S$ & 120 & 1,2 \\
\hline * & n mEst & 1.0 & 0.1 \\
\hline , & 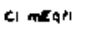 & 747 & 1.3 \\
\hline 4 & N & 2,09 & 2,03 \\
\hline 3 & $\begin{array}{l}\mathrm{CO}_{2} \cos \alpha \\
\text { mEast }\end{array}$ & 42,4 & 2,2 \\
\hline 3 & ke mg or & 8,7 & a \\
\hline J & $\begin{array}{l}\text { PRA } \\
\text { mimish }\end{array}$ & +60,3 & $* \geqslant 0$ \\
\hline
\end{tabular}

\begin{tabular}{|c|c|c|c|}
\hline$n$ & OMine & $\bar{x}$ & E.s. \\
\hline s & Ne mEqIZA $\mathrm{k}$ & 40,0 & 43 \\
\hline a & 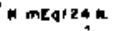 & 230 & 1,0 \\
\hline , & c. $m e d / 24 n$. & 5,3 & 4,7 \\
\hline 2 & H+H & 2,74 & $0, m$ \\
\hline a & $\begin{array}{l}\text { Aldosterena } \\
\text { megras m }\end{array}$ & $+\infty$ & a. \\
\hline$n$ & $\begin{array}{l}\text { velumen } \\
\text { mo/mg/24 } \mathrm{h}\end{array}$ & 101,4 & 73 \\
\hline & & & \\
\hline
\end{tabular}

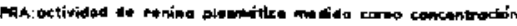

do angiolomina I $\mathrm{Ne} 1,07: 0,43 \mathrm{mg} / \mathrm{m} / \mathrm{m}$.

\section{SINDROME DE BARTTER INFUSION DE ANGIOTENSINA II}

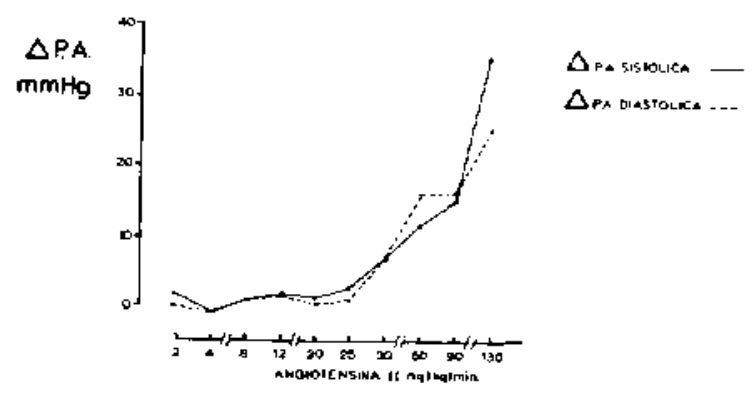

\section{INFUSION DE NORACRENALINA $\mathrm{ng} / \mathrm{kg} / \mathrm{min}$.}

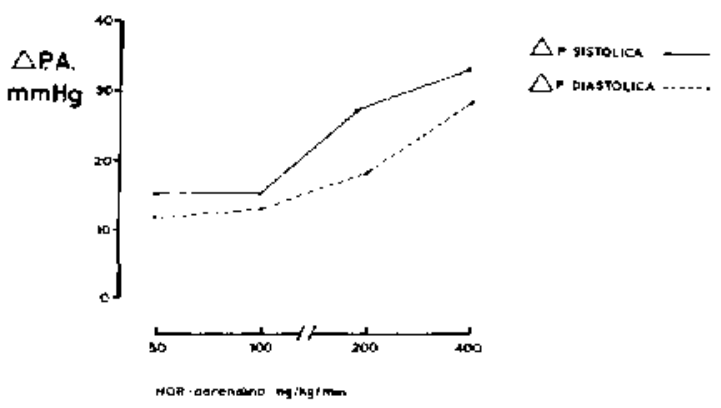

Figura 1. El alza de $20 \mathrm{Imm} \mathrm{Hg}$ en la presión diastólica se ohservó al intundir l35 $\mathrm{ng} / \mathrm{kg} / \mathrm{min}$ de A II y $400 \mathrm{ng} /$ $\mathrm{kg} / \mathrm{min}$ de noradrenalina $(\mathrm{N}=<10 \mathrm{n} / \mathrm{kg} / \mathrm{min}$ para $\mathrm{A}$ I [ $y<50 \mathrm{ng} / \mathrm{kg} / \mathrm{min}$ para noradrenalina).

table (Na $50 \mathrm{mE} q /$ día, K $30 \mathrm{mEq} / \mathrm{día}$ ) se procedió a la sobre hidratación leve de la paciente con 
infusión de suero glucosado al $5 \%$ hasta obtener un volumen urinario de $2 \mathrm{ml} / \mathrm{min}$. En estas condiciones se midió la reabsorción fraccional distal de cloro, según la fónmula $\mathrm{C}_{\mathrm{H}_{2} \mathrm{O}} / \mathrm{C}_{\mathrm{H}_{2} \mathrm{O}}+\mathrm{C}_{\mathrm{Cl}}{ }^{10}$ donde $\mathrm{C}_{\mathrm{H}_{2} \mathrm{O}}=$ depuración agua libre y $\mathrm{C}_{\mathrm{Cl}}=$ depuración de cloro. El resultado obtenido fue de 0.57 , siendo lo normal 0.65 o nás. ${ }^{11}$ La biopsia renal efectuada por lumbotomía derecha, demostró hiperplasia del aparato yuxtaglomerutar en el $30 \%$ de los glomérulos observados (Figura 2).

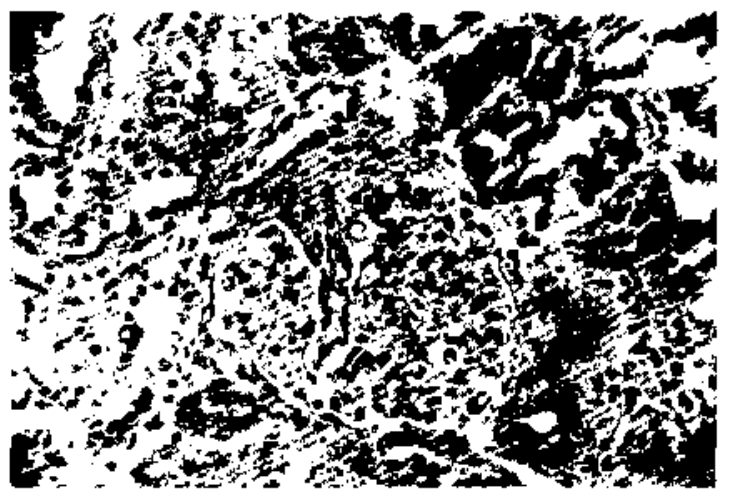

Figura 2. Hiperplasia del aparato yuxtanglomerular.

\section{DISCUSION}

En esta paciente lo más llamativo fue la hipokalemia severa, mantenida y refractaria a la suplementación con potasio en altas dosis, con sus consecuencias de alcalosis metabólica, hiper-reninemia y síndrome poliúrico.

La presión arterial nomal permitió descartar desde el comienzo el hiperaldosteronismo primario, lo cual fue reafirmado por la presencia de hiperreninemia. Asimismo, la alcalosis metabólica persistente hizo descartable la acidosis tubular renal. Los antecedentes clínicos descartaron también la posibilidad de ingesta crónica de diuréticus o los vómitos subrepticios, situación esta última en que la excreción urinaria de cloto es mínima a diferencia de lo observado en nuestra paciente, ${ }^{12}$ que presentaba marcada clonuresis.

El estudio demostró además que la pérdida de potasio ocuria en este caso por vía urinaria: potasio urinario $23,8 \pm 1,9 \mathrm{mEg} / 24 \mathrm{~h}(\mathrm{n}=8)$, para una ingesta de nenos de $5 \mathrm{mEq} / \mathrm{kg} /$ dia y en presencia de kalemias inferiores a $2.0 \mathrm{mEq} / \mathrm{l}$.

En un sujeto nomal, el potasio urinario proviene principalmente de secreción por las células del túbulo distal. El potasio filtrado se reabsorbe casi - completamente en el túbulo proximal mientras que en el asa de Henle su reabsorción es pasiva y depende de la reabsorción de cloro y sodio.

Es así como, trente a toda pérdida exagerada de potasio por el riñón, debe procederse a examinar la función del túbulo distal y del asa de Henle.

Entre las tubulopatías que pueden acompañarse de hipokalemia está la llamada "nefropatía perdedora de sal", que puede corresponder a una etapa en la evolución de la nefritis crónica en que la destrucción tubular afecta parcialmente a los receptores celulares para aldosterona. En estos casos se produce hiponatremia con elevación de aldosterona la que al actuar sobre los receptores restantes puede inducir kaliuresis.

Las deficiencias de magnesio también pueden próducir pérdida renal de potasio. ${ }^{13}$

Por último hay algunos casos descritos de tubulopatias con degeneración de las células del túbulo distal ${ }^{14,15}$, 16 o asociados a estigmas somát$\cos ^{17}$ que pueden llevar a hipokalemia. Ninguna de estas se acompaña de hiperplasia del aparato yuxtaglomerular que si bien es inespecífica, es indispensable para el diagnóstico del síndrome de Bartter. Nuestra paciente, en cambio, adenás de presentar marcada hiperplasia del aparato yuxtaglomerular, no presentaba fenómenos degenerativos tubulares ni distorsión parenquimatosa renal, como tampoco hipomagnesemia ni hiperaldosteronismo exagerado. Todo esto permite razonablemente descartar en ella la presencia de una tubulopatia perdedora de potasio como las mencionadas.

En cambio, la función del asa de Henle se encontraba claramente alterada. La reabsurción de cloro, que es un buen marcador del funcionamiento de este segmento del nefrón, se encontraba deprimida tal como se ve en el síndrome de Bartter. ${ }^{10,11}$

Al deprimirse la reabsorción de cloro en el asa de Henle, deja de reabsorberse tambiên sodio y potasio. Todo esto aumenta el flujo de electrolitos $y$ agua al túbulo distal con el que se intensifica la reabsorción de sodio y se agrega secreción de potasio; esto explica que el sodio urinario pueda bajar mucho y en cambio el potasio urinario no pueda disminuir como correspondería a un sujeto con hipokalemia.

La hipokalemia mantenida produce mayor déficit de potasio. Es posible que ésta sea también la causa de la hiper-producción de prostaglandinas ${ }^{1 / 5}$ y de brandikinina ${ }^{19}$ que se ve en el síndrome de Bartter lo que explica la falta de respuesta vascu- 
lar al efecto presor de angiotensina II y noradrenalina ${ }^{20}$ como lo observamos en nuestra paciente.

En resumen, pensamos que el defecto de la reabsorción de cloro demostrado en esta paciente permite explicar la secuencia fisiopatológica de sus signos y sintomas (Figura 3 ), configurando as $i^{\circ}$ el diagnóstico de síndrome de Bartter.

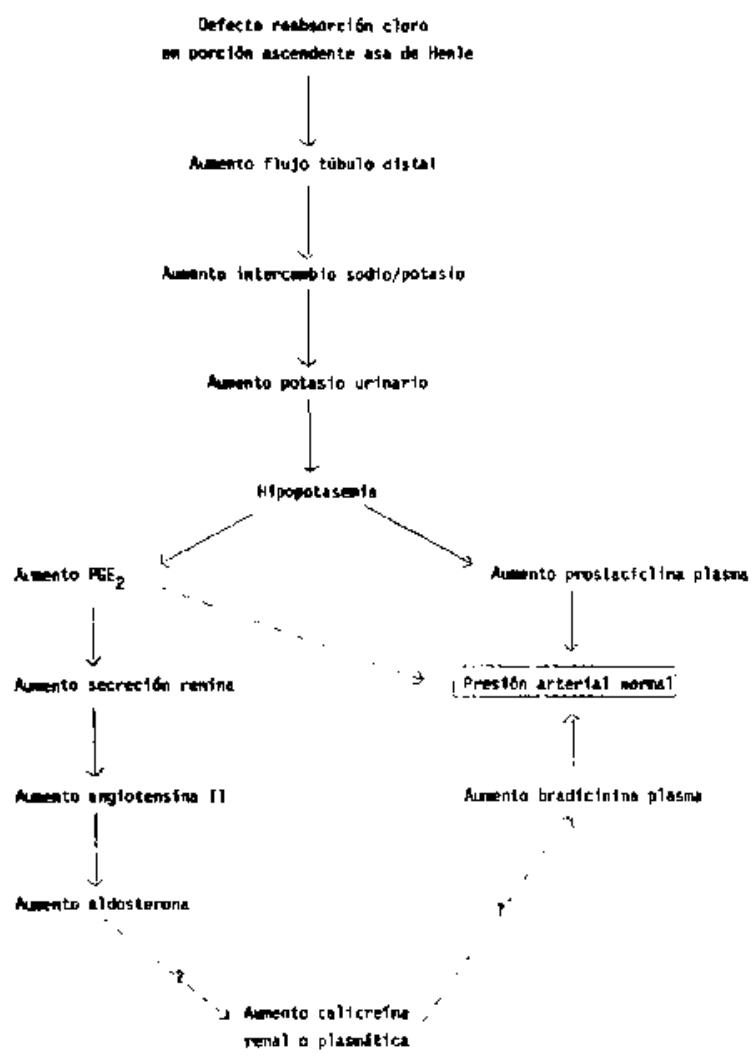

Figura 3. Secuencia fisiopatologrica de signos y sintomas del síndrome de Bartter, explicudos a partir de un defecto en la reabsorción de cloro en la porción ascendente del asa de Henle, como el comprobado en esta paciente. Las líneas punteadas indican los pasos para los que todavia no hay suficiente eviderıcia experimental.

Consideramos que la comprobación de dicho defecto en un paciente con las características clínicas y bioquímicas del síndrome restringe el nombre de "síndrome de Bartter" sólo a aquellos en quienes se pueda así explicar la causa de su afección. Por el contrario, si dicho defecto no se puede demostrar, deberán azgotarse los esfuerzos por llegar al diagnóstico histológico y funcional de otra nefropatia de las que pueden dar un cuadro similar.

\section{FESUMEN}

Se presenta el caso de una pre-escolar de 4 años con hipokalemia grave y persistente, alcalosis metabólica, hiper-reninemia con hiperplasia del aparato yuxtaglomerular y presión arterial normal. Se efectuó un estudio sistemático de las posibles causas de hipokalemia. La comprobación de un defecto en la reabsorción fraccional distal de cloro, como se describe en el síndrome de Bartter, permite explicar razonablemente la secuencia fisiopatológica de sus signos y sintomas.

\section{AGRADECIMIENTOS}

Los autores agradecen al Dr. Guillemo Murray, Aruátomo-Patólogo, Universidad de Chile, por el detallado estudio morfológico renal. También agradecen a la señorita Gladys Solari, Enfermera Jefe del Servicio de Pediatrúta del Hospital Paula Jaraquemada, por su cooperación al estudio.

\section{REFERENCIAS}

'Cunningham, R.J.; Bronhand, B.H.; Petrusick, T. y Iravts, L.B. I ong-tern use of propranolol, ibuprofen and spiromblactone in the marlagement of Bartter's symdrome. Pediat. 63: 754-756, 1979.

${ }^{2}$ Donker, A.J.M. Indomethacin in Barter's syndrome. Nephron 19: 200.1977.

"Dibon, M.d.; Shah, V. y Mitchell, D. Burtter's syndrome: 10 cases in childhoud. Results of long-term indomethacin therapy. Quart. J. Medicine, New Series XLVIHI, 191: 424-446, 1979.

${ }^{4}$ Banter, F.C.; Pronove, P.: Gill, J.R.; Moc Cordle, R C. Hyperplitsia of the juxtaglomerular complex with hyperaldosteronism and hypokalemic alkalosis. Am. J. Mell 33: 811-828, 1962 .

${ }^{5}$ Gitl, J.R. Jr.; Barter, F,C.; Taylor, A.A.- Radiar, N. Intpaired tulyular chloride reabsorption as a proximal cause of Bartter's svndrome. Clin. Res. 25; 526A, 1977.

'Godoy, R; Paros, MA.; Loper, J.M. Validación de un método radioinmunológico para culantíficación de aldasterona urinarid. Libro de Resúmenes de las VI Jomadas Cientíticas del Area Biologica, Universidad Católica de Chile. Santiago, Octubre, 1.980 , p. 59.

'Silber, R.H. y Porter, C.C. The determinutions of 17,21 dihydroxy-20 ketosteroids in urine aud plasma. J. Biol. Chent. 210: 923, 1954

${ }^{8} Z$ Zimmermann, V. Eine larbreaktion ales sexual honnune und ihre Anwendung zur quantitative colorimetrischen Bestimung. Iloppe Seyler Z. Physical Chem. 233: 25T, 1935.

"Kapion, N.M. y Silah, J.G. The effect of angsictensin II un hlocm pressure in humans with hypertensive disease. J Clin. Invest. 43: 659, 1964.

"Gill, J.R. Jr, y Bartler, F.C. Evidenee for a prostaglandin-itydependent defect in chloride reabsorption in the lon of He'nle as a proximal cunse of Bartter's syndrone. An. J. Mied. 65: 766-772, 1978.

" Rodniguez, J.A.; Siragy, H.M.; Fox R.F.: Detea, C.S. y Bartter, F.C. Effectiveness of fractional distal chloride reabsorption in the diagnosis of Battter's stmdrome. Clin. Res. 28: $845 \mathrm{~A}, 1960$. 
12 Veldhuis, J.D.: Bandin, C.W. y Demers, L.M. Metabolic mimicty of Bartter's syndrome by covert vomiting. Am. J. Med, 66: 361. 363, 1979.

${ }^{13}$ Shls, M.E. Experimental human magnesium depletion. Medicine 48:61, 1869 .

14 Gullner, H.G.; Gill, d.R.; Barter, F.C. Chan, J.C.M. y Dichonan, P.S. A fanilial disorder with hypokallemic alkalosis, hyperrenj. nernia, aldosteronism, high urinary prostaglandins and normal blood pressure that is not "Hartter's syndrome". Trans. An. Pbysicians 92: 175, 1979.

15 Rosenbaum, P. y Hughes, M. Persistent, probably congenitad, hypokalemic metabolic alkalosis with hyaline degeneration of renal tubules and nonnal urinary aldosterone. AMA J. Dis. Child. 96: 560, 1958.

16 Slater, RJ.; Azzopandi, P.; Skater, P.E y Chute, A.L An unusual case of chronic hypokalemia assuciated with renal tubular degeneration. Am. J. Dis. Child. 96: 469-470, 1958.

${ }^{17}$ Tokedo, R.; Morimoto, S.; Kuroda, M.; Murakmi, M. Renal tubular acidosis presenting as a syndrome resembling Bartter's syndrome in a patient with arachnodactyly. Acta Endocrinol. 73: $531-542,1973$.

${ }^{18}$ Galves, O.G.; Bay, W.H.; Roberts, B.W. y Ferris, T.F. The hemodynamic effects of potassium deliciency in the dog. Circulation Res. 4): suppl. 1, pp. 11-16, 1977.

${ }^{1 Y}$ Vind, J.M.; GIt. J.R. Jr; Bouden, R.E.; Ptsuno, J.J.; lro, J.L.: Radfor, N.; Toylor, A.A.; Zusman, R.N.; Bartter, F.C. y Keiser, H.R. The kallikrein-kinill system in Bartter's syndrome and its response to prostaglandin synthetase inhibitors. J. Clin. [nvest. 6]: 1671-1682, 1978.

20 Vane, J.R.: Mc Giff, J.C. Possible contributions of endogenous prostaglandins to the control of blood pressure. Circulation Res. 36: suppl. I. pp, 68-75, 1975. 